\title{
Scaffold hopping to percept the achiral compound and functionalizing on target protein Plasmepsin II
}

\begin{abstract}
Malaria is a vector-borne infectious disease caused by protozoan parasites of the genus Plasmodium. Four types of the plasmodium parasite can infect humans; the most serious forms of the diseases are caused by Plasmodium falciparum. Plasmepsin group of enzymes are key enzymes in the life cycle of malarial parasites. Plasmepsin II enzyme is responsible for the formation of the hemozoin through the hemoglobin degradation pathway. Specification of potential drug target by inhibiting Plasmepsin II Enzyme, N-(R-CarboxyEthyl)-Alpha-(S)-(2-Phenylethyl) presently used as an inhibitor, to prevent the degradation. Changeover of side chains based on isomeric property of Achiral compounds is aimed to be a new inhibitor for the Plasmepsin II enzyme. Scaffold hopping is an approach used to discover new chemical classes by replacing a portion (the scaffold) of a known compound, while preserving the remaining chemical groups, under the assumption that they are important for biological activity. The main goal of this investigation was to find out the types of combination with the ligand to be the most active in the inhibition of Plasmepsin II enzyme, among the Achiral compounds. Achiral inhibitors revealed exploitation of a new inhibitor observed in Plasmepsin II protein, by Electrostatic potential, binding energy, and bond interactions.
\end{abstract}

Keywords: malaria, plasmepsin II, hemoglobin degradation pathway, achiral compounds, scaffold hopping
Volume 5 Issue 5 - 2017

\section{Suganya Selvaraj, Murugesh Easwaran, Praveen Kumar, Nilavuamuthan Chandrasekar, Ruban Durairaj, Radha Vadivelu, Shanmughavel Piramanayagam Department of Bioinformatics, Bharathiar University, India}

Correspondence: Shanmughavel Piramanayagam, Associate Professor, Computational Biology Lab, Department of Bioinformatics, Bharathiar University, India, Tel 9486446 I0I, Email shanmughavel@buc.edu.in

Received: October 31, 2016 | Published: May 30, 2017
Abbreviations: TLR, toll-like receptor; PM II, plasmepsin II; HTS, high throughput screening

\section{Introduction}

Malaria is a vector-borne disease caused by protozoan parasites of the genus Plasmodium i.e. Plasmodium vivax, Plasmodium ovale, Plasmodium falciparum and Plasmodium malariae. Only these four types of the plasmodium parasite can infect humans as partial prophylaxis infection; the most serious form of disease caused by Plasmodium falciparum. During infection, Plasmodium merozoites invade and then replicate within red blood cells. During the log phase within the host cell, the parasite obtains nutrients by taking up and then digesting hemoglobin within an acidic food vacuole. The digestion of hemoglobin releases monomeric $\alpha$-hematin (ferriprotoporphyrin IX). Released compound predicting its hypertoxicity property, since it is a pro-oxidant and it catalyzes the production of reactive oxygen species. Oxidative stress is believed to be generated during the conversion of heme to hematin. ${ }^{1}$ Free hematin can also bind and then the disrupt cell membranes, damaging cell structures and causing the lysis of the host erythrocyte. Reporting a novel mechanism that the host utilizing Toll-like receptor (TLR) 9 to recognize Plasmodium DNA, which may be a prior induction of fever during the replicative, process (Lag Phase) disease. These findings reveal an important mechanism of disease patho-physiology that may also apply to other microbial diseases. ${ }^{2}$ It also corrects previous findings claiming that hemozoin is a direct TLR9 stimulus and refines them by showing that hemozoin itself important for presenting the DNA to TLR9 but does not stimulate the receptive process (Figure 1). Although it is too early to predict how these findings will influence the development of future malaria treatment options, it is likely that it will open new pathways of interference with the malaria fever reaction, and this may influence the course of disease. ${ }^{3,4}$



Figure I Illustrates the potential mechanism of malaria-induced fever, shows that hemozoin contains plasmodial DNA and that it "presents" or internalizes DNA. Plasmodial DNA then intracellularly interacts with TLR9, initiating signal transduction.

A new series of plasmepsin II (PM II) inhibitors has been prepared based on 4-aminopiperidine-tert-butyl-carbamate. These theoretically analysed compounds might be coursed for anti malarial drugs acting via a new mechanism, and therefore be less susceptible to parasite resistance now often observed with current anti malarial therapies. Among the various targets, the hemoglobin degradation pathway- 
involving several parasite specific enzymes like the plasmepsins and the falcipains is of major interest. One possibility to strike against the parasite is to inhibit an enzyme within this pathway interrupting the nutrition source and therefore eliminating the parasite by starvation. PM II has been chosen because its inhibition process follows a completely new and promising pathway to fight against the malaria parasite having a good potential of being devoid of fast emerging resistance (Figure 2). This target fits well within our core competencies in aspartic protease inhibitor as a therapeutic strategy, which has been proven especially proficified in the field of HIV-protease inhibition to treat AIDS. The lead compounds depicted below emerged from a High Throughput Screening (HTS) of a commercial library containing 50,000 compounds for PM II inhibitory activity. ${ }^{5}$

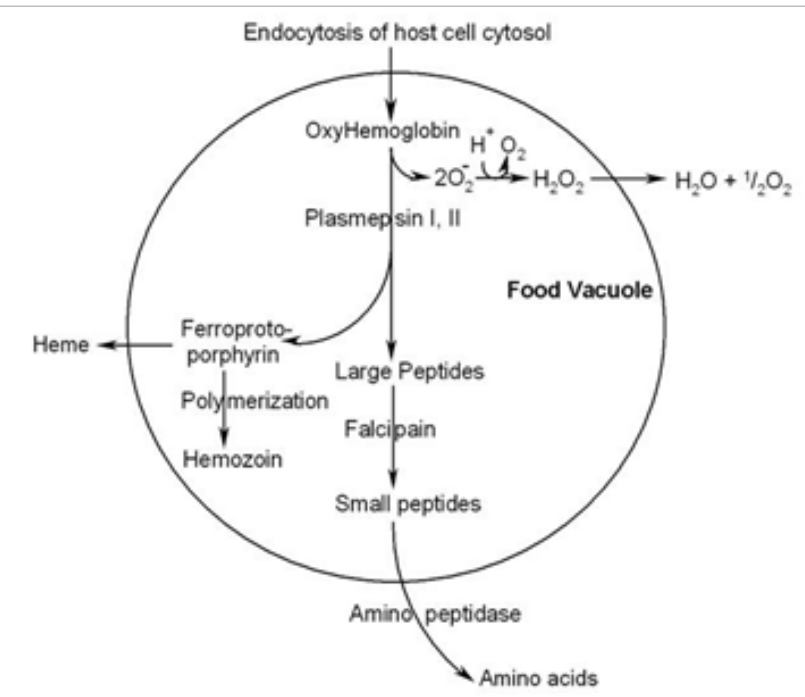

Figure 2 Simplified overview of a process in the food vacuole and shows many targets for drug development.

Resistance to current drugs such as chloroquine and mefloquine is spreading at an alarming rate, and our antimalarial armamentarium is almost depleted. Crystal structure of recombinant plasmepsin II complexes with pepstatin A. first reported from P. falciparum. The crystals contain molecules in two different conformations, revealing a remarkable degree of inter domain flexibility of the enzyme. The structure was used to design a series of selective low molecular weight compounds that inhibit both Plasmepsin II and the growth of $P$. falciparum in culture. ${ }^{6}$ The main criterion of emergence is spreading of resistant parasite strains. Essentials of new treatments are indigenous fact to control over the disease. Discovering new potential targets towards this goal are likely to be more successful. Such efforts discoveries will result in new drugs that are functionally and structurally different from the existing drugs and therefore will overcome existing resistances. Here we have focused on the aspartic protease Plasmepsin II, which is a promising new drug target. ${ }^{7}$

Several x-ray structures of PM II have been described previously, but thus far, structure-based drug design has been hampered by the fact that only inhibitors comprising a statine moiety or derivatives thereof have been published. Our drug discovery finds innovative and easily synthesized inhibitors against aspartic proteinase yielded some high

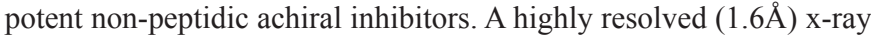
structure of PMII is presented, featuring a potent achiral inhibitor in an unprecedented orientation, contacting the catalytic aspartates indirectly via the "catalytic" water. Major side chain rearrangements in the active site occur, which open up a new pocket and allow a new binding mode of the inhibitor. Moreover, a second inhibitor molecule could be located unambiguously in the active site of PMII. ${ }^{8}$ A threedimensional (3D) quantitative pharmacophore model was developed from a training set of structurally diverse substituted areas against Raf-1 kinase, which was well validated to be highly predictive by two methods, namely, Test set prediction and Cat-Scramble method. Then a virtual database searching was performed with the pharmacophore model as a 3D query, and the bioactivities of the retrieved hits were predicted by the Pharmacophore. The study facilitates the discovery and rational design of novel lead compound with potential inhibitory activity targeting Raf-1kinase. ${ }^{9,10}$

A new extension to the method for structure-based design is reported that uses the shape of the target site as an additional constraint. This enables the docking process, for example in library design and virtual screening, to be quantified in terms of how many, and which; pharmacophoric hypotheses can be matched by a compound or a library of compounds. ${ }^{11}$ A scaffold hopping approach has been exploited to design a novel class of cannabinoid (CB1) receptor antagonists for the treatment of obesity. On the basis of shape-complementarity and synthetic feasibility the central fragment, a methylpyrazole, in Rimonabant was replaced by a pyrazine. The synthesis and CB1 antagonistic activities of a new series of 5, 6-diaryl-pyrazine-2-amide derivatives are described. Several compounds showed antagonist potency below $10 \mathrm{nM}$ for the $\mathrm{CB} 1$ receptor. $^{12}$

\section{Materials and methods}

Plasmepsin II (www.rcbs.org/pdb/1LF4) structure was retrieved from Protein data bank. This protein structure was generated by removing water molecules. Hydrogen atoms were then added to the protein. Minimization of energy were performed (using universal force field $)^{13,14}$ in the presence of restraints since the removal of hetero atoms, at the same time maintaining the protein conformation very close to that observed in the crystallographic model. The resulting receptor model was saved to a PDB file (compatible with both Argus Lab input file formats).

\section{Preparation of Receptor}

Although many structures are available for the receptor along with the ligand bound to it. Without the ligand, the PDB structure (ID 1LF4) was download and saved it as (*.pdb) (Figure 3A). Heteroatom was removed to get receptor structure.

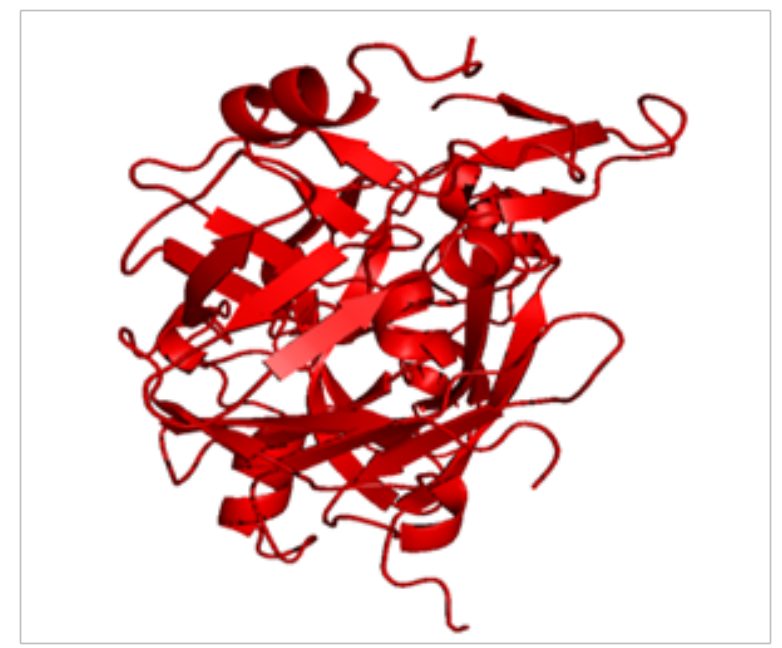

Figure 3A X-ray crystal structure of Plasmepsin II. 


\section{Preparation of ligand}

What if server (http://swift.cmbi.ru.nl/servers/html/index.html) was used to predict the active site of the protein. This active site was inspected to make suitable docking site (Figure 3B). Ligand was collected from Pubchem compound CID657149 (www.ncbi.nih.gov) (Figure 4A), input structure was generated with chemsketch and saved it in mol format (Figure 4B). Open Babel (http://openbabel.org/wiki/ get-open-babel) tool which is used to convert the molecular structures into desired form, saved it in PDB file format. Achiral compound was taken to develop inhibitors of Plasmepsin II allowing for the use of a parallel chemistry setup for the fast preparation of small focused libraries. Using Argus Lab (www.arguslab.com) docking tool the binding site was again defined from the coordinates of the ligand in the original PDB file. Argus dock exhaustive search docking engine was used, with grid resolution of $0.40 \AA$. Docking precision was set to 'high precision' and 'flexible ligand docking' mode was employed for each docking run and results are evaluated in Table $1 .{ }^{15}$ Resulting analysis was done based on criteria like Analysis based on docking energy scoring; Placement quality of the ligand inside the binding pocket of the target protein; Interactions between the ligand and target protein; Inter Atomic Distance; In PyMOL select preset option to view the ligand interaction. From the wizard option select measurement to find out the inter atomic energy results (hydrogen bond interaction).



Figure 3B Dot surface (pink color) represents the binding site of Plasmepsin II was inspected to make suitable docking site using active site prediction tool



Figure 4A Structure of Ligand. Structure of N-(R-Carboxy-Ethyl)-Alpha-(S)(2-Phenylethyl) compound without any substitutions.
Table I Comparison of the protein-ligand (Achiral) docking efficiencies.

\begin{tabular}{llll}
\hline Achiral & $\begin{array}{l}\text { Bonded } \\
\text { Protein } \\
\text { Residues }\end{array}$ & $\begin{array}{l}\text { Inter } \\
\text { Atomic } \\
\text { Distance(A) }\end{array}$ & $\begin{array}{l}\text { Docking } \\
\text { Energy(kcal/ } \\
\text { mol) }\end{array}$ \\
\hline $\begin{array}{l}\text { Methyloxyl and } \\
\text { 2-Methyl-IH- } \\
\text { imidazole }\end{array}$ & GLN I2 & 2.22 & \\
$\begin{array}{l}\text { THR I 19 } \\
\text { 2-methyl and } \\
\text { imidazole }\end{array}$ & VAL I60 & 3.33 & -9.38792 \\
$\begin{array}{l}\text { 3-Methyl phenol } \\
\text { and 3-Methyl } \\
\text { Pyridine }\end{array}$ & GLU 74 & 3.37 & \\
$\begin{array}{l}\text { 4-Methyl phenol } \\
\text { and 4-Methyl } \\
\text { Pyridine }\end{array}$ & TYR 192 & 2.86 & -15.8167 \\
$\begin{array}{l}\text { 2-Methyl butane } \\
\text { and ethyl methyl } \\
\text { ether }\end{array}$ & VAL 78 & 2.7 & \\
\hline
\end{tabular}

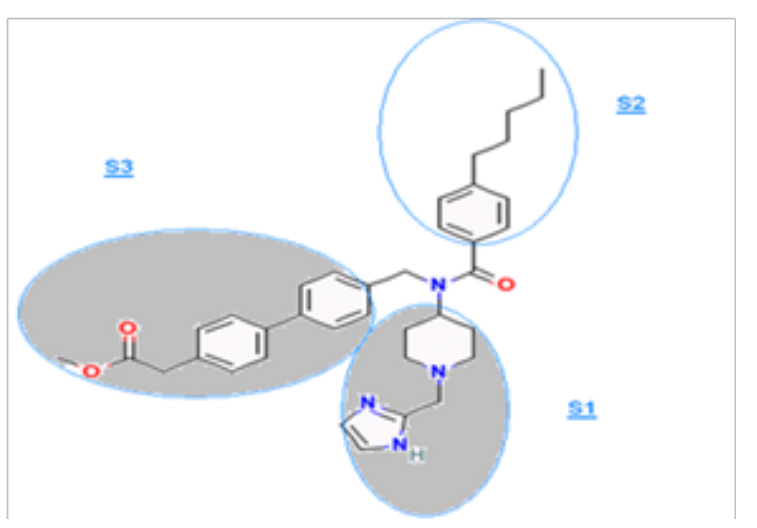

Figure 4B Core structure of the Achiral compound have the Substitution position of SI, S2, and S3.

\section{Results and discussion}

PDB structure of Plasmepsin II-ID 1LF4 was downloaded without inhibitor and used for docking (Figure3A). Core structure of the Achiral compound have the Substitution position of S1, S2, and S3 (Figure 4B) for each analog are listed in Tables $2 \& 3$. It has been shown that Achiral serves as a template to develop inhibitors of PM II allowing for the use of a parallel chemistry setup for the fast preparation of small focused libraries (Table $2 \& 3$ ). S1 and S3 regions were taken for the pharmacophore mapping to predict the compound activity. S1 and S3 side chains were substituted with Methane, hydroxyl, Methyloxyl, 2 methyl pentane, 2-methyl-1H-imidazole, 3-ethyl furan of the ligand for docking purpose ${ }^{16}$ And each of the modified ligands was docked with the Plasmepsin II enzyme. In an effort to establish efficient docking routines for computational screening of compound on protein structures, Plasmepsin II has been selected and a variety of docking options and scoring functions were compared. These included rigidbody and flexible docking and scoring based on force field energy. Methyl and 2-methyl-1H-imidazole substituted achiral compound 
forms hydrogen bond interacting with the residue THR 217, TYR 77, GLY 80 of Plasmepsin II enzyme with the inter atomic distance $2.86 \AA, 2.70 \AA, 3.35 \AA$ with respectively and the least docking energy$15.8167 \mathrm{kcal} / \mathrm{mol}$. Methyloxyl, 2 methyl pentane substituted achiral compound forms hydrogen bond interacting with the residues ASP 19, GLN 12, THR 119, Val $1602.22 \AA, 3.33 \AA, 3.53 \AA$ of Plasmepsin II enzyme with the inter atomic distance $2.22 \AA, 3.33 \AA, 3.53 \AA$, $3.37 \AA$ with respectively and the least docking energy-9.38792kcal/ mol. Methyloxyl, 3-ethyl furan substituted achiral compound forms hydrogen bond interacting with the residue ASP 10 of Plasmepsin II enzyme with the inter atomic distance $3.18 \AA$ with respectively and the least docking energy- $10.3754 \mathrm{kcal} / \mathrm{mol} .{ }^{17}$

Table 2 Contains the details of binding energy between the side chains modified Achiral ligand and Plasmepsin II. Unknown: There is no interaction between protein and ligand molecule.

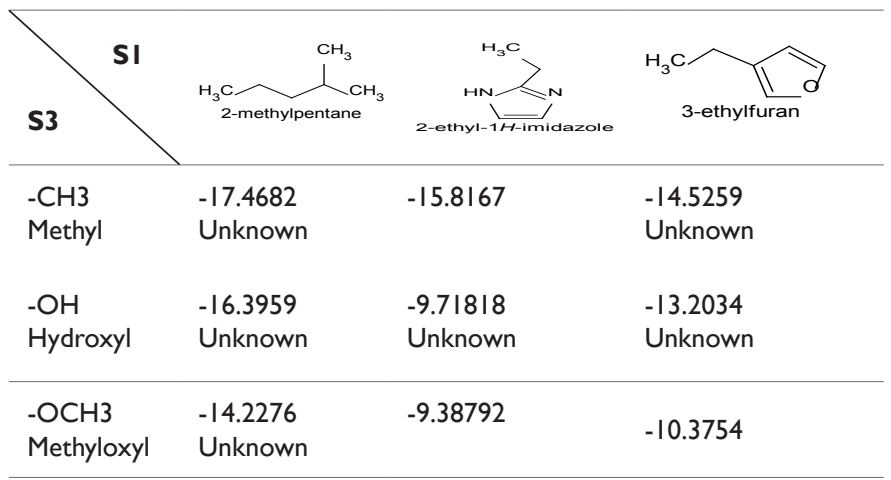

Table 3 Contains the details of docked energies between the side chains modified Achiral ligand and Plasmepsin II. Unknown: There is no interaction between protein and ligand molecule.

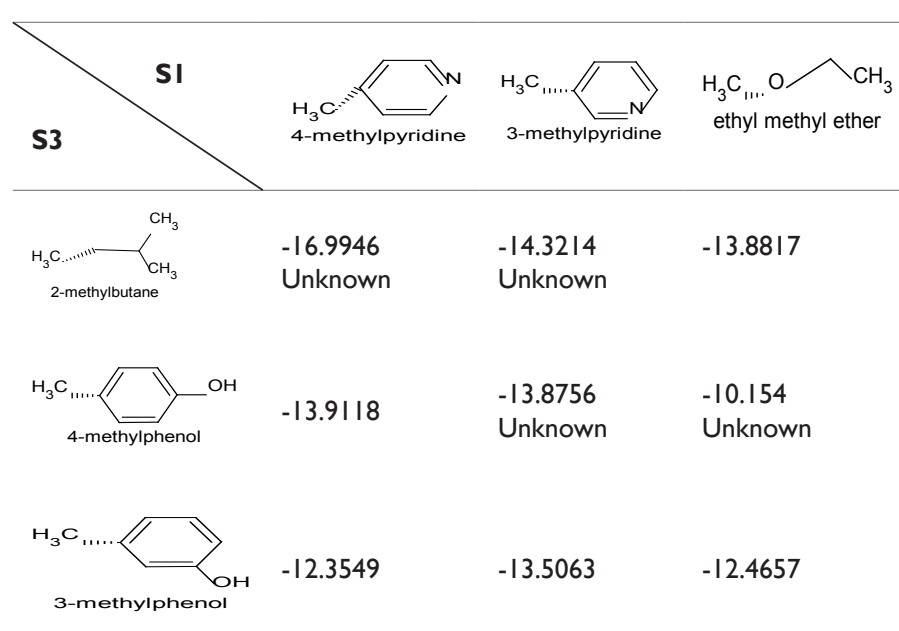

Docking was done with the S1 and S3 side chains modifications of the drug. These regions are substituted with, 4 methyl pyridine, 3-methyl pyridine, ethyl methyl ether, 2-methyl butane, 4-methyl phenol and 3-methyl phenol respectively. 2-methyl butane and ethyl methyl ether substituted achiral compound forms hydrogen bond interacting with the residue VAL 78 of Plasmepsin II enzyme with the inter atomic distance $3.34 \AA$ and the least docking energy$13.8817 \mathrm{kcal} / \mathrm{mol}$. 4-methyl phenol and 3-methyl pyridine substituted achiral compound form hydrogen bond interacting with the residue ASP 130 of Plasmepsin II enzyme with the inter atomic distance $2.21 \AA$ and the least docking energy-13.9118kcal $/ \mathrm{mol}$. 3-methyl phenol and 4-methyl pyridine substituted achiral compound forms hydrogen bond interacting with the residue LEU 33 of Plasmepsin II enzyme with the inter atomic distance $4.32 \AA$ and the least docking energy- $12.3549 \mathrm{kcal} / \mathrm{mol}$. 3-methyl phenol and 3-methyl pyridine substituted achiral compound form hydrogen bond interacting with the residues GLU 74, TYR 192 of Plasmepsin II enzyme with the inter atomic distance $3.05 \AA, 3.22 \AA$ and the least docking energy$13.5063 \mathrm{kcal} / \mathrm{mol}$. 3-methyl phenol and ethyl methyl ether substituted achiral compound form hydrogen bond interacting with the residue LEU 33 of Plasmepsin II enzyme with the inter atomic distance $2.46 \AA$ and the least docking energy- $12.4657 \mathrm{kcal} / \mathrm{mol}$.

It has been procedurized by taking nearly 18 compounds for post docking analysis study against the Plasmepsin II were performed. Modification of side shows variety of complex structures. From this theoretical experiment, the suitable ligand which has more interacting hydrogen bonds and which has least docking energy score were selected as the inhibitor for the Plasmepsin II enzyme. The Table 1 shows the list of ligands has the number of hydrogen bonds and the least docked energy score with the Plasmepsin II enzyme. Most of the inter-atomic distance is formed in the nitrogen and oxygen atoms. Nitrogen atom of 2-ethyl-IH-imidiazole form hydrogen bonds interacting with the residues ASP 19, GLN 12, VAL 160 of Plasmepsin II protein with the inter atomic distances $2.22,3.33,3.37 \AA$ respectively and also oxygen atom forms single hydrogen bond interacting with THR 119 of protein with the inter atomic energy $3.53 \AA$ (Figure 5). Through the docking procedure 2-ethyl-IH-imidiazole Achiral compound with Plasmepsin II the least binding energy has been calculated and the value is- $9.38792 \mathrm{kcal} / \mathrm{mol}$. Nitrogen atom of the 2-ethyl-IH-imidiazole form hydrogen bonds interacting with the residues TYR 77, GLY 80 with the inter atomic distances $2.86,2.70 \AA$ with respectively and also oxygen atom forms hydrogen bond interacting with the residue THR 217 of Plasmepsin II protein with the inter atomic distance $3.35 \AA$ (Figure 6). Through the docking procedure 2-ethyl -IH-imidiazole Achiral compound with Plasmepsin II the least binding energy has been calculated and the value is- $15.8167 \mathrm{kcal} / \mathrm{mol}$.

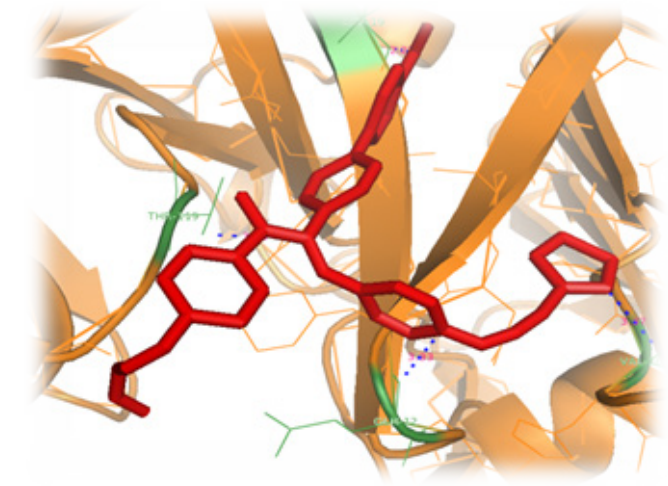

Figure 5 Structure of Plasmepsin II (orange color) complexed with Achiral (red color) which is substituted with Methyloxyl and 2-methyl-IH-imidazole at the areas of SI and S3. Blue color line indicates the four hydrogen bond interaction with the Plasmepsin II protein residues.

Oxygen atom of 3-Methyl phenol and 3-methyl pyridine forms single hydrogen bond interacting with the TYR 192 residues of Plasmepsin II with the inter atomic distance $3.22 \AA$ (Figure 7). Through the docking procedure 3-methyl pyridine compound with Plasmepsin II the least binding energy has been calculated and the value is- $13.5063 \mathrm{kcal} / \mathrm{mol}$. Oxygen atom of 2 Methyl butane forms single hydrogen bond interacting with the residue ASP 130 of the Plasmepsin II protein with the inter atomic distance $2.21 \AA$ (Figure 8). Through the docking of the 2 Methyl butane Achiral compound 
with Plasmepsin II the least docked energy is calculated and the value is- $13.9118 \mathrm{kcal} / \mathrm{mol}$. Nitrogen atom of 2 Methyl butane and Propan1 -amine forms single hydrogen bond interacting with the residue VAL 78 of the Plasmepsin II protein with the inter atomic distance $3.34 \AA$ (Figure 9). Through the docking of the 2 Methyl butane Achiral compound with Plasmepsin II the least docked energy is calculated and the value is- $13.8817 \mathrm{kcal} / \mathrm{mol}$.

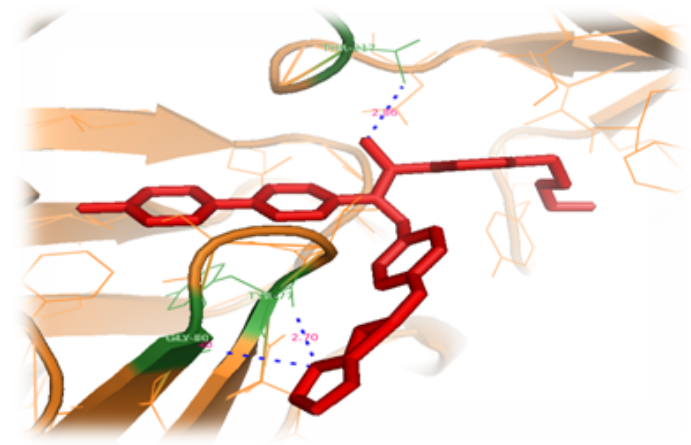

Figure 6 Illustrates the Complex of Plasmepsin II (orange color) with ligand (red color) which is substituted with Methyl and 2-methyl-IH-imidazole at the areas of SI and S3. Two Hydrogen bonds are represented by lines (Blue color) which accommodate binding site.



Figure 7 Structure of PIm II orange color-coded, a molecule of achiral, shown in a green color representation, is at the binding cleft. The active site residues (green color), interacting with, side chains substituted with 3-methyl phenol and 3-methyl pyridine. Nitrogen atoms of 3-methyl pyridine forms single hydrogen bond interacting with the residue GLU 74 and inter atomic distance $3.05 \AA$.

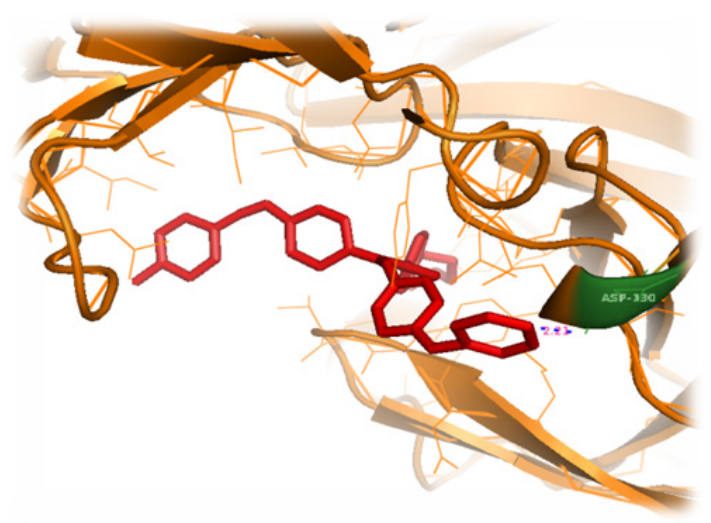

Figure 8 Complex structure of Plasmepsin II (orange color) and Achiral (red color) which is substituted with 3-methyl phenol and methyl benzene at the regions of SI and S3. One Interacting hydrogen bond was shown in blue color.

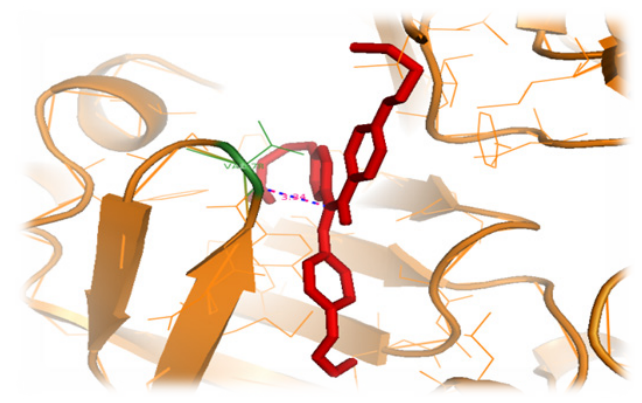

Figure 9 Inhibitor (red color) substituted with 2 methyl butane and propane-I-amineat the regions of SI and S3, which was docked with the structure of Plasmepsin II (orange color). One interacting hydrogen bond with the Plasmepsin II is shown in blue color.

\section{Conclusion}

In the recent trends anti malarial drugs such as quinine or artemisinin are coursed. However, parasites have evolved to be a resistant to many of these drugs. The hemoglobin degradation enzymes of malaria parasites have been validated as targets for drug development and its discoveries. Of these enzymes, only the aspartic proteases, called Plasmepsins, have been produced recombinant and structurally characterized. Achiral inhibitors revealed exploitation of a new inhibitor observed in Plasmepsin II protein by its binding energy value and its inhibitory constant. Finally, 18 hits were identified as potential leads against Plasmepsin, which exhibited good estimated activities, high docking scores, similar binding mode to experimentally proven compounds and favorable drug-like properties. Mathematical biology concept in space complementary algorithm for docking procedure reveals Methyloxyl, 2-ethyl-IH-imidiazole acts as the best inhibitor against Plasmepsin II enzyme. Proceedings of drug development for the malarial fever by using Achiral compounds can be achieved from Protein engineering and wet lab techniques in pharma industry. Conclusion should elucidate how the results communicate to the theory presented as the basis of the study and provide a concise explanation of the allegation of the findings.

\section{Acknowledgements}

None.

\section{Conflict of interest}

The author declares no conflict of interest.

\section{References}

1. Jiang S, Prigge ST, Wei L, et al. New Class of Small Nonpeptidyl Compounds Blocks Plasmodium falciparum Development In Vitro by Inhibiting Plasmepsins. Antimicrobial Agents and Chemotherapy. 2001;45(9):2577-2584.

2. Dow GS, Koenig LM, Wolf L, et al. The Antimalarial Potential of 4 Quinolinecarbinolamines May Be Limited due to Neurotoxicity and Cross-Resistance in Mefloquine-Resistant Plasmodium falciparum Strains. Antimicrobial Agents and Chemotherapy. 2004;48:2624-2632.

3. Schumann RR. Malarial fever: Hemozoin is involved but Toll-free. Proc Natl Acad Sci. 2007;104(6):1743-1744.

4. Chauhan VS, Bhardwaj D. Current Status of Malaria Vaccine. Bio Drugs. 2003;84:143-182.

5. Mueller R, Huerzeler M, Boss C. Synthesis of Plasmepsin II InhibitorsPotential Antimalarial Agents. Molecules. 2003;8(7):556-564. 
6. Silva M, Lee AY, Gulnik SV, et al. Structure and inhibition of plasmepsin II, A hemoglobin-degrading enzyme from Plasmodium falciparum. Proc Natl Acad Sci. 1996;93(19):10034-10039.

7. Boss C, Bildstein SR, Weller $\mathrm{T}$, et al. Inhibitors of the Plasmodium falciparum parasite aspartic protease plasmepsin II as potential antimalarial agents. Curr Med Chem. 2003;10(11):883-907.

8. Prade L, Jones AF, Boss C, et al. X-ray Structure of Plasmepsin II Complexed with a Potent Achiral Inhibitor. $J$ Biol Chem. 2005;280:23837-23843.

9. Lia HF, Lua T, Zhua T, et al. Virtual screening for Raf-1 kinase inhibitors based on pharmacophore model of substituted ureas. European Journal of Medicinal Chemistry. 2008;44:1240-1249.

10. Kenakin T. Quantifying Biological Activity in Chemical Terms: A Pharmacology Primer to Describe Drug Effect, Biological Reagents and Assay Development. ACS Chem Biol. 2009;4(4):249-260.

11. Mason JS, Cheney LD. Library design and virtual screening using multiple 4-point pharmacophore fingerprints. Pacific Symposium on Biocomputing. 2000;5:573-584.

12. Bostrom JJ, Berggren K, Elebring T, et al. Scaffold hopping, synthesis and structure-activity relationships of 5,6-diaryl-pyrazine-2-amide derivatives: A novel series of CB1 receptor antagonists. Bio org Med Chem. 2007;15(12):4077-4084.
13. Oda A, Takahashi O. Validation of Argus Lab Efficiencies For Binding Free Energy Calculations. Chem-Bioinformatics Journal. 2009;9:52-61.

14. Karkolaa S, Richmonda SA, Wahala K. Pharmacophore modelling of 17 $\beta$-HSD1 enzyme based on active inhibitors and enzyme structure Laboratory of Organic Chemistry. Molecular and Cellular Endocrinology. 2008;301(25):225-228.

15. Marriott DP, Dougall IG, Meghani P, et al. Lead generation using pharmacophore mapping and three-dimensional database searching: application to muscarinic $\mathrm{M}(3)$ receptor antagonists. J Med Chem. 1999;42(17):3210-3216.

16. Wade RC, Qrtiz AR, Gago F. Comparative Binding Energy Analysis, In 3D QSAR in Drug Design: Ligand Protein Interactions and Molecular Similiarity and Perspectives. Drug Discovery and Design. 1998;9:19-34.

17. Westling J, Cipullo P, Hung SH, et al. Active site specificity of plasmepsin II. Protein Science. 1999;8(10):2001-2009. 